\title{
CRESCIMENTO E ABSORÇÃO DE FÓSFORO EM PLANTAS DE Eucalyptus grandis ASSOCIADAS A FUNGOS MICORRÍZICOS EM DIFERENTES DOSES DE FÓSFORO E POTENCIAIS DE ÁGUA do SOLO ${ }^{(1)}$
}

\author{
M. F. FERNANDES(2), H. A. RUIZ(3), \\ J.C. L. NEVES ${ }^{(4)} \&$ R. M. C. MUCHOVEJ (5)
}

\begin{abstract}
RESUMO
Avaliaram-se a produção de parte aérea (MS), a absorção (CP) e a eficiência de utilização de fósforo (EUP) por plantas de Eucalyptus grandis inoculadas com Glomus etunicatum (Ge) e, ou, Pisolithus tinctorius (Pt), em diferentes combinações entre doses de $\mathbf{P}$ e potenciais hídricos do solo $(\psi)$, utilizando-se técnica de raízes subdivididas em vasos geminados, em casa de vegetação. Um dos vasos do conjunto gemi nado continha os tratamentos em arranjo fatorial de duas doses de P (P60 e P120, correspondentes a adições de 60 e $120 \mathrm{mg} \mathrm{dm}^{-3} \mathrm{de}$ solo), três $\psi(-10,-40$ e $-300 \mathrm{kPa}$ ) e quatro tratamentos com inóculos fúngi cos (não inoculado, Ge, Pt, Ge + Pt). Outro vaso do conjunto continha 4,5 litros de solução nutritiva, sem $P$. I ndependentemente da dose de $P$ adicionada, a colonização por Ge foi reduzida com a diminuição do $\psi$. Quanto à colonização por Pt, observouse ausência de resposta a $\psi$ em P60 e aumento em P120. A colonização ectomi corrízica foi reduzida quando Pt foi inoculado concomitantemente a Ge. Aumentos do CP, em função do incremento do $\psi$, foram observados em P60 e P120. No entanto, MS e E UP responderam positivamente à elevação de $\psi$ apenas em P120. A inoculação isolada com Ge não apresentou efeito sobre as plantas, nas combinações entre $\psi$ e P. A colonização por Pt apresentou efeitos diferenciados sobre as plantas, dependendo das condições de $\psi$ e P no solo. Na combinação $\mathrm{P} 60$ e -300 kPa, correspondente à situação de menor disponibilidade de $\mathbf{P}$, observou-se efeito deletério da colonização por $\mathrm{Pt}$, isolada ou concomitantemente com Ge, sobre a EUP das plantas. Efeitos positivos da inoculação isolada com Pt sobre o CP foram observados nas combinações entre $P 120$ e $\psi$ de -10 kPa e-40 kPa, embora apenas na segunda situação este maior CP tenha sido acompanhado de incremento da MS.
\end{abstract}

Termos de indexação: micorriza, fósforo, eucalipto.

\footnotetext{
(1) Parte da Tese de M estrado do primeiro autor apresentada à Universidade F ederal de Viçosa - UFV. Recebido para publicação em outubro de 1998 e aprovado em abril de 1999.

(2) Pesquisador da Embrapa Tabuleiros Costeiros. CEP 49025-040 Aracaju (SE).

(3) Professor do Departamento de Solos, UFV. CEP 36571-000 Viçosa (MG). Bolsista do CNPq.

(4) Professor do Departamento de Solos, UFV.

(5) Professora do Southwest Florida Research and Education Center. Immokalee, FL 34142-9515 (EUA).
} 
SUMMARY: GROWTH AND PHOSPHORUS UPTAKE BY Eucalyptus grandis SEEDLINGS ASSOCIATED TO MYCORRHIZAL FUNGI IN DIFFERENT PHOSPHORUS RATES AND SOIL WATER POTENTIALS

\begin{abstract}
Shoot growth, phosphorus uptake (PC) and utilization efficiency (PUE) of Eucalyptus grandis seedlings associated to Glomus etunicatum (Ge) and, or, Pisol ithus tinctorius (Pt), in different $\mathrm{P}$ rates and soil water potentials $(\psi)$, wereevaluated in a greenhouseexperiment with two gemi nated pots, using a split-root technique. One of the pots in the geminated set contained the treatments, arranged in a factorial scheme of two $\mathrm{P}$ rates (P60 and $\mathrm{P} 120$, corresponding to additions of 60 and $120 \mathrm{mg} \mathrm{dm}^{-3}$ soil), thre $\psi(-10,-40$ and $-300 \mathrm{kPa})$ and four mycorrhizal inoculation treatments (without inoculation, with $\mathrm{Ge}$, with $\mathrm{Pt}$, and with $\mathrm{Ge}+\mathrm{Pt}$ ). The other pot did not contain $\mathrm{P}$ in its nutrient solution. Regardless of the $\mathrm{P}$ rate added in soil, colonization decreased in responseto $\psi$ reductions. This $\psi$ reduction resulted in no changes on Pt infection in P60, but led to a significant increase in P120. The Pt col onization was lower when it was inoculated with Gethan when inoculated al one. Increases on $\mathrm{PC}$ in responseto $\psi$ increment were observed in both $\mathrm{P}$ rates. However, shoot dry matter (DM) and PUE showed a positiveresponseto $\psi$ increment only at P120. Therewas no effect of Geinoculation separately on theplants, in any $\psi$ and $P$ ratecombinations. Pt col onization presented distinct effects, depending on thesecombinations. At P60 and $-300 \mathrm{kPa}$ condition, in which P availability was very low, a deleterious effect of Pt inoculation, separately or associated with Ge, was observed on PUE. Pt inoculation, separately, presented a positive effect on PC in the combinations between P120 and $\psi-10 \mathrm{kPa}$ or $-40 \mathrm{kPa}$, but only in the second situation therewas a DM increase
\end{abstract}

Index terms: mycorrhiza, phosphorus, Eucalyptus.

\section{NTRODUÇÃO}

O fósforo é transportado na solução do solo essencialmente por difusão (Bouldin, 1961; Olsen et al., 1962), sendo a velocidade desse transporte regulada por diversos fatores, destacando-se: oteor de água no solo, a interação do $\mathrm{P}$ e os colóides do sol o ea distância a ser percorrida pel o nutriente até atingir as raízes das plantas (Bhadoria et al., 1991a,b,c). As variações na difusão do P, provocadas pelas modificações desses fatores, alteram o fornecimento do nutriente para as plantas. Deacordo com Bhadoria et al. (1991a,b), a umidade do solo apresenta relação di reta com a vel ocidade de difusão do P no solo. Para Ruiz et al. (1988), à medida quea umidade do sol o aumenta, ofil me deágua queenvol ve as partículas torna-se mais espesso, diminuindo as interações dos íons e a superfície do solo e, conseqüentemente, aumentando a concentração de fosfato dissolvido em solução.

Além dos fatores que determinam a velocidade de difusão do fosfato no solo, as características morfológicas e fisiológicas das raízes também são fatores determinantes para a aquisição de $P$ pelas plantas. Dentre as características morfológicas de importância para a absorção desse nutriente, destacam-se o número e o comprimento dos pêlos radiculares e a taxa de crescimento das raízes (Barley, 1970; I toh \& Barber, 1983; Rheinheimer et al., 1997). O incremento dessas características promove a maior exploração do sol o e a redução da distância a ser percorrida pel o nutrienteem difusão (Clarkson, 1985).

As associações micorrízicas representam uma estratégia de aumento da absorção de $\mathrm{P}$ pelas plantas, já que as hifas desses fungos incrementam o volume de solo explorado pelas raízes (Rhodes \& Gerdemann, 1975), podendo absorver e translocar para a planta íons fosfatos localizados muito além da área de ação dos pêlos radiculares (Clarkson \& Hanson, 1980). Alguns autores relatam ainda que características fisiológicas relacionadas com a vel ocidade e com a eficiência de absorção de P pelas raízes são modificadas quando estas estãoassociadas a fungos micorrízicos (Cress et al., 1979; Faquin et al., 1990). Além disto, de acordo com N elsen \& Safir (1982a), a forte interação do $P$ e as superfícies de componentes da fase sólida do solo, principalmente em condições de umidade que limitam a difusão deste nutriente, pode ser atenuada pel o transporte de $\mathrm{P}$ internamente às hifas, após sua absorção pelo fungo associado à raiz. Dessa forma, grandes distâncias podem ser percorridas pelo íon sem contato com os colói des do solo. A importância desse transporte via hifa pode ser expressiva, visto que comprimentos de $80 \mathrm{~cm}$ ou mais de hifas de fungos micorrízicos por $\mathrm{cm}$ de raiz de cebola já foram relatados por Sanders \& Tinker (1973). 
Embora as espécies de plantas, em sua maioria, associem-se a fungos micorrízicos arbusculares ou ectomicorrízicos, as pertencentes ao gênero Eucalyptus, dentre outras, formam micorrizas de ambos os tipos (Chilvers et al., 1987).

O objetivo deste trabal ho foi avaliar os efeitos da colonização micorrízica por Glomus etunicatum e, ou, Pisol ithus tinctorius no crescimento e absorção de $P$ por Eucalyptus grandis, em diferentes combinações entre potenciais da água do solo e doses de P.

\section{MATE RIAL E MÉTODOS}

O sol outilizado foi col etado na camada de $0-20 \mathrm{~cm}$ de profundidade, em área sob vegetação de Pinus sp. da Universidade Federal deViçosa, Viçosa (MG), sendo classificado como L atossolo Vermel ho-Amarelo álico, textura muito argilosa. O solo foi seco ao ar, passado através de peneira com malha de $4 \mathrm{~mm}$ de abertura para o ensaio em casa de vegetação e de $2 \mathrm{~mm}$ para a sua caracterização química e física (Quadro 1). Amostras desse solo foram incubadas com uma mistura de $\mathrm{CaCO}_{3}$ e $\mathrm{MgCO}_{3}$, na proporção de 4:1 em moles, em quantidade necessária para elevar o $\mathrm{pH}\left(\mathrm{H}_{2} \mathrm{O}\right)$ para 5,8. Concomitantemente, incorporaram-se doses de 60 ou $120 \mathrm{mg} \mathrm{dm}^{-3}$ de $\mathrm{P}$, usando $\mathrm{CaHPO}_{4}$. Após 60 dias de incubação, em condições de aerobiose, com umidade próxima a $-40 \mathrm{kPa}$, as amostras foram secas ao ar. Novas determinações de $\mathrm{P}$ pelo extrator Mehlich-1 indicaram valores de 4 e $10 \mathrm{mg} \mathrm{dm}^{-3}$, respectivamente. As amostras de solo receberam, então, duas fumigações, com intervalo de $72 \mathrm{~h}$ entre elas, utilizando-se brometo de metila na dosagem de $90 \mathrm{~cm}^{3} \mathrm{~m}^{-3}$ de solo.

Cada unidade experimental foi constituída por dois vasos, com capacidade de $5 \mathrm{dm}^{3}$, montados em forma geminada (Ruiz et al., 1988). Em um dos compartimentos, colocaram-se 4,5 $\mathrm{L}$ de solução nutritiva de Clark (1975) modificada quanto à concentração de ferro $\left(0,08 \mathrm{mmol} \mathrm{L}^{-1}\right)$ e livre de $\mathrm{P}$, com vistas a fornecer os demais nutrientes e água de forma não-restritiva ao crescimento das plantas. Esse compartimento recebeu arejamento constante e foi recoberto com tampa de isopor revestida com papel alumínio. A solução nutritiva teve seu pH ajustado, diariamente, para 5,5, sendo renovada a cada sete dias. O segundo compartimento recebeu $4,2 \mathrm{dm}^{3}$ desol o com os diferentes tratamentos, segundo um esquema fatorial ( $22 \times 3 \times 2)$, correspondendo a quatro combinações de inóculos dos fungos micorrízicos Glomus etunicatum (Ge) e Pisolithus tinctorius (Pt): sem inóculo, Ge, Pt e Ge + Pt, três potenciais hídricos $(\psi)(-10,-40$ e $-300 \mathrm{kPa})$ e duas doses de $\mathrm{P}$ (60 e $120 \mathrm{mg} \mathrm{dm}^{-3}$ ). Utilizou-se o delineamento em blocos casualizados, com quatro repetições e duas plantas por vaso.

\section{Quadro 1. Características físicas e químicas da amostra do Latossolo Vermelho-Amarelo da região de Viçosa (MG)}

\begin{tabular}{lc}
\hline \multicolumn{1}{c}{ Característica } & Valor \\
\hline Areia grossa (\%) ${ }^{(1)}$ & 18 \\
Areia fina (\%) ${ }^{(1)}$ & 12 \\
Silte (\%) ${ }^{(1)}$ & 6 \\
Argila (\%) ${ }^{(1)}$ & 64 \\
Densidade do solo $\left(\mathrm{g} \mathrm{cm}^{-3}\right)^{(2)}$ & 1,09 \\
Retenção de água $\left(\mathrm{g} \mathrm{kg}^{-1}\right)$ & \\
$-10 \mathrm{kPa}$ & 312 \\
$-40 \mathrm{kPa}$ & 260 \\
$-100 \mathrm{kPa}$ & 256 \\
$-300 \mathrm{kPa}$ & 239 \\
$-500 \mathrm{kPa}$ & 231 \\
$\mathrm{pH}$ em água $(1: 2,5)$ & 4,7 \\
Complexo sortivo $\left(\mathrm{cmol}_{\mathrm{c}} \mathrm{dm}^{-3}\right)$ & \\
$\mathrm{A} 1^{3+}(3)$ & 2,0 \\
Ca & 0,1 \\
$\mathrm{Mg}^{2+}(3)$ & 0,0 \\
$\mathrm{~K}^{2+(4)}$ & 0,05 \\
$\mathrm{P}$ disponível $\left(\mathrm{mg} \mathrm{dm}^{-3}\right)^{(4)}$ & 1 \\
Carbono orgânico $\left(\mathrm{g} \mathrm{dm}^{-3}\right)^{(5)}$ & 19,0 \\
\hline
\end{tabular}

(1) Método da pipeta (EMBRAPA, 1997); (2) Método da proveta (EMBRAPA, 1997); ${ }^{(3)}$ Extrator KCl 1 mol L-1 ${ }^{(4)}$ Extrator Mehlich-1; (5) Método Walkley-Black (J ackson, 1958).

As plantas de E. grandis foram obtidas de sementes desinfestadas com água oxigenada a 30\%, por 10 min, lavadas em água estéril e germinadas em areia autoclavada. A pós 20 dias da semeadura, procedeu-se à primeira sel eção das mudas, por critério de uniformidade. As selecionadas tiveram suas raízes pivotantes cortadas a um centímetro do coleto para estimular o desenvolvimento de raízes secundárias. As mudas foram logotransferidas para caixas plásticas que continham $8 \mathrm{~L}$ de solução nutritiva de Clark (1975), meia força, mas com a concentração de ferro em $0,08 \mathrm{mmol}^{\mathrm{L}-1}$ e mantidas sob aeração constante, por 30 dias.

Previamente ao transplantio, instalaram-se tensiômetros a 2/3 da profundidade dos vasos nos tratamentos de-10 e-40 kPa, para o monitoramento da umidade do solo. Na superfície desses compartimentos, colocaram-se camadas de areia lavada de $1 \mathrm{~cm}$ de espessura para reduzir as variações na umidade do solo. A umidade desses vasos foi corrigida, diariamente, pela manhã, após a leitura dos potenciais nos tensiômetros. Os tensiômetros foram escorvados todos os dias após o pôr do sol. Nos vasos com $-300 \mathrm{kPa}$, a irrigação foi realizada apenas por ocasião da instalação do experimento. A quantidade de água adicionada aos vasos para atingir este potencial foi determinada pela curva característica de retenção deágua nosolo. Para minimizar perdas de água dos vasos deste tratamento, aumentou-sea espessura da camada de 
areia para 1,5 cm e cobriu-se o compartimento com uma tampa de isopor revestida com papel alumínio (Ruiz et al., 1988).

As plantas foram novamente selecionadas e transplantadas para os vasos geminados, tomando-se ocuidado de distribuir as raízes omais uniformemente possível entre os dois compartimentos.

A inoculação com o fungo mi corrízico arbuscular (FMA) Ge foi feita no transplantio, utilizando-se inóculo misto com cerca de 1.260 esporos por vaso. O fungo ectomicorrízico Pt, isolado $90 \mathrm{~A}$, foi inoculado cinco dias após o plantio, com uma seringa, sob a forma de suspensão micelial. A massa seca micelial adicionada a cada vaso foi de aproximadamente 18,3 mg. Após 20 dias do transplantio, foram real izadas inocul ações de reforço com os fungos. N os tratamentos com Ge, incorporousea mesma quantidade de inóculo misto em um sulco de $1 \mathrm{~cm}$ de profundidade $5 \mathrm{~cm}$ de raio ao redor das plantas, tomando-se o cuidado de não romper as raízes. Na inoculação de reforço de Pt, adicionaramse, em um sulco idêntico ao descrito para o inóculo de $\mathrm{Ge}$, seis discos de meio MNM de $6 \mathrm{~mm}$ de diâmetro, contendo mi cél io de Pt retirado das bordas de colônias com 25 dias de idade. Para evitar a influência dos veículos dos inócul os sobre a resposta das plantas, adições de discos de meio MNM sem Pt foram feitas nos tratamentos que não receberam este tipo de inóculo com micélio, ao passo queincorporações de inóculo misto autoclavado de Ge foram feitas nos tratamentos que não receberam o inóculo viável deste FMA.

O experimento foi coletado 80 dias após o transplantio para os vasos geminados. A parteaérea foi cortada a $1 \mathrm{~cm}$ do coleto, seca em estufa de circulação forçada a $65^{\circ} \mathrm{C}$, por $72 \mathrm{~h}$, pesada e triturada em moinho com peneira de malha de $0,25 \mathrm{~mm}$. As raízes cresci das no compartimento com sol o foram lavadas em água corrente para remoção do solo. Em seguida, amostras de cerca de $2 \mathrm{~g}$ de raízes finas foram retiradas earmazenadas em uma solução F.A.A. (5 mL deformol, $5 \mathrm{~mL}$ deácido acético e $90 \mathrm{~mL}$ de ál cool). Essas amostras foram tratadas de acordo com a técnica proposta por Koske \& Gemma (1989) para coloração das estruturas fúngicas. Após a coloração, procedeu-se à determinação da percentagem do comprimento radicular colonizado por GeePt, utilizando o método das interseções em placa reticulada, proposto por Ambler \& Young (1977) e testado por Giovannetti \& Mosse (1980). Duzentos miligramas da parte aérea de plantas de cada vaso foram mineralizados por digestão nítrico-perclórica, obtendo-se os extratos para a determinação dos teores deP por colorimetria (Braga \& Defelipo, 1974). A eficiência de utilização deP (EUP) foi cal culada pela relação entrea matéria seca e o teor do nutriente (Siddiqi \& Glass, 1981).

Os dados foram submetidos à análise de variância com desdobramento dos graus de liberdade para tratamentos, demodoa avaliar, para todas as variáveis estudadas, o efeito do $P$, bem como o efeito linear e o quadrático do $\psi$ dentro de cada dose desse nutriente. Para comparar os tratamentos fúngicos, foram utilizados contrastes ortogonais distintos, de acordo com a variável avaliada. Para estudar o efeito da presença de um fungo sobre a capacidade de col onização do outro, os contrastes utilizados foram os seguintes:

C1:[(Ge) - (col onização concomitante por GeePt)] e C2:[(Pt - colonização concomitante por Ge e Pt)]. Os contrastes utilizados para comparar os tratamentos fúngicos quanto a seus efeitos sobre as demais variáveis foram: $\mathrm{C} 1:[(3 \times$ controle não inoculado) - (Ge +Pt + colonização concomitante por Ge e Pt)]; C2:[(Ge) - (Pt)] e C3: [(2 x colonização concomitante por Ge e Pt) - (Ge + Pt)].

Para avaliar as características entre os três tratamentos que receberam inóculo fúngico com o controle não inoculado, dentro de cada uma das combinações entre $\mathrm{P}$ e $\psi$, procedeu-se ao teste de Dunnett a 5\%.

\section{RESULTADOS E DISCUSSÃO}

\section{Colonização mi corrízica}

A col onização radicular por G. etunicatum (Ge) e $P$. tinctorius (Pt) diminuiu com o incremento da dose deP (Quadros 2 e3). Relações inversas entre o estado nutricional de plantas de diferentes espécies e a intensidade de colonização radicular por fungos micorrízicos arbusculares (Mosse, 1973; Sanders, 1975) eectomi corrízicos (Marx et al., 1977; Dixon et al., 1981) já foram extensivamente relatadas na literatura.

Independentemente da dose de $\mathrm{P}$, a redução do $\psi$ diminuiu a colonização micorrízica por Ge (Quadros 2e3). Deacordo com Koske(1981), esporos de Gigaspora gi gantea incubados por sete dias em areia, nos potenciais de 0 (zero), -500 e $-1.000 \mathrm{kPa}$, obtidos pela adição de diferentes quantidades de pol ietilenoglicol, apresentaram cerca de 95,50 e 5\% degermi naçãoe comprimento de tubos germinativos de $1 \mathrm{~cm}, 2$ e $1 \mathrm{~mm}$, respectivamente. A germinação de esporos de Glomus epigaeum também foi incrementada pela elevação do potencial hídrico do solo (Daniels \& Trappe, 1980). No entanto, diferentemente do observado neste estudo, resultados evidenciaram a ausência de efeito da redução da umidade do sol o sobre a colonização de raízes de Triticum aestivum cv. Anza e Carthamus tinctorius cv. S555 por G. etunicatum, conformeBryla \& Duniway (1997).

As plantas dos tratamentos Pt e Ge + Pt, aval iadas conjuntamente, apresentaram aumento da colonização por Pt, em resposta à redução do $\psi$, em 
Quadro 2. Percentagem do comprimento radicular colonizado por Glomus etunicatum (Ge) e Pisolithus tinctorius (Pt), considerando as doses de fósforo, os potenciais da água do solo e os tratamento fúngicos

\begin{tabular}{cclrr}
\hline Fósforo Potencial & $\begin{array}{c}\text { Tratamento } \\
\text { fúngico }\end{array}$ & Ge & Pt \\
\hline $\mathrm{mg} \mathrm{dm}^{-3}$ & $\mathrm{kPa}$ & & $\overline{80}$ & \\
60 & -10 & $\mathrm{Ge}$ & 80,2 & 2,2 \\
& & $\mathrm{Pt}$ & 0,0 & 59,0 \\
& & $\mathrm{Ge}+\mathrm{Pt}$ & 82,2 & 17,5 \\
& -40 & $\mathrm{Ge}$ & 79,5 & 0,0 \\
& & $\mathrm{Pt}$ & 0,0 & 68,5 \\
& -300 & $\mathrm{Ge}+\mathrm{Pt}$ & 80,7 & 21,2 \\
& & $\mathrm{Ge}$ & 64,0 & 1,2 \\
& & $\mathrm{Pt}$ & 1,0 & 63,7 \\
120 & -10 & $\mathrm{Ge}+\mathrm{Pt}$ & 68,5 & 21,0 \\
& & $\mathrm{Ge}$ & 60,5 & 1,0 \\
& & $\mathrm{Pt}$ & 0,0 & 39,0 \\
& -40 & $\mathrm{Ge}+\mathrm{Pt}$ & 55,7 & 12,5 \\
& & $\mathrm{Ge}$ & 55,0 & 0,0 \\
& & $\mathrm{Pt}$ & 1,5 & 32,7 \\
& -300 & $\mathrm{Ge}+\mathrm{Pt}$ & 40,5 & 19,7 \\
& & $\mathrm{Ge}$ & 41,7 & 0,0 \\
& & $\mathrm{Pt}$ & 0,0 & 54,5 \\
& & $\mathrm{Ge}+\mathrm{Pt}$ & 43,2 & 25,0 \\
\hline
\end{tabular}

P120; ao passo que, em P60, não foram observados efeitos significativos do $\psi$ na colonização por Pt (Quadros 2e3). Embora, segundo Mexal \& Reid (1973), diversas espécies de fungos ectomicorrízicos tenham apresentado crescimento máximo em meios de cultura submetidos a potenciais de $-500 \mathrm{a}-1.500 \mathrm{kPa}$, acredita-se que, no presente estudo, o efeito do potencial sobre a colonização tenha sido indireto, pela alteração da disponibilidade de $\mathrm{P}$ para as plantas, visto que este efeito dependeu da dose de $P$ adicionada ao sol o. Ou seja, a ini bição da col onização por Pt, em resposta ao incremento de $\psi$, em P120, pode ter sido conseqüência da mel horia da nutrição fosfatada das plantas. Em P60, o gradiente do conteúdo de P (CP) entre os $\psi$ foi menor queem P120, (Quadros 4 e 5), podendo ter sido insuficiente para causar alterações na colonização da raiz.

A formação de ectomicorrizas nos tratamentos de inoculação concomitante com as duas espécies fúngicas foi menor do que nos de inoculação isolada com Pt. Entretanto, a col onização MA não foi afetada pela presença do fungo ectomicorrízico (Quadros 2 e3). Competição entrefungos MA eectomicorrízicos, em uma mesma raiz, foi sugerida por Chilvers et al. (1987). Esses autores relataram que as MA, predominantes, inicialmente, no sistema radicular de Eucalyptus dumosa, foram substituídas, mais tarde, pelas ectomicorrizas. Chilvers et al. (1987) demonstraram ser a predominância inicial das micorrizas arbusculares resultante da maior

Quadro 3. Análise de variância da percentagem do comprimento radicular colonizado por Glomus etunicatum $(\mathrm{Ge})$ e Pisolithus tinctorius $(\mathrm{Pt})$, considerando as doses de fósforo (P), os potenciais da água do solo $(\psi)$ e os tratamentos fúngicos ${ }^{(1)}$

\begin{tabular}{|c|c|c|c|c|c|}
\hline \multicolumn{3}{|c|}{ Glomus etunicatum } & \multicolumn{3}{|c|}{ Pisolithus tinctorius } \\
\hline$F V^{(1)}$ & GL & QM & $\mathrm{FV}$ & GL & QM \\
\hline Blocos & 3 & 158,94 & Blocos & 3 & 34,77 \\
\hline $\mathrm{P}$ & 1 & $8586,75^{* *}$ & & 1 & $1496,33^{* *}$ \\
\hline$\psi(L) d / P(60)$ & 1 & $900,00 *$ & $\psi(L) d / P(60)$ & 1 & 68,06 \\
\hline$\psi(Q) d / P(60)$ & 1 & 216,75 & $\psi(Q) d / P(60)$ & 1 & 111,02 \\
\hline$\psi(L) d / P(120)$ & 1 & $976,56 *$ & $\psi(L) d / P(120)$ & 1 & $812,25 *$ \\
\hline$\psi(Q) d / P(120)$ & 1 & 67,69 & $\psi(Q) d / P(120)$ & 1 & 234,08 \\
\hline$C 1 d / P(60) d / \psi(-10)$ & 1 & 8,00 & $C 2 d / P(60) d /{ }_{\psi}(-10)$ & 1 & $3528,00 * *$ \\
\hline $\mathrm{C} 1 \mathrm{~d} / \mathrm{P}(60) \mathrm{d} / \psi(-40)$ & 1 & 2,88 & $C 2 d / P(60) d / \psi(-40)$ & 1 & $4474,58^{* *}$ \\
\hline $\mathrm{C} 1 \mathrm{~d} / \mathrm{P}(60) \mathrm{d} / \psi(-300)$ & 1 & 40,50 & $\mathrm{C} 2 \mathrm{~d} / \mathrm{P}(60) \mathrm{d} / \psi(-300)$ & 1 & $3646,58^{* *}$ \\
\hline$C 1 d / P(120) d / \psi(-10)$ & 1 & 46,08 & $C 2 d / P(120) d / \psi(-10)$ & 1 & $1404,50 * *$ \\
\hline $\mathrm{C} 1 \mathrm{~d} / \mathrm{P}(120) \mathrm{d} / \psi(-40)$ & 1 & 420,00 & $\mathrm{C} 2 \mathrm{~d} / \mathrm{P}(120) \mathrm{d} / \psi(-40)$ & 1 & 338,00 \\
\hline $\mathrm{C} 1 \mathrm{~d} / \mathrm{P}(120) \mathrm{d} / \psi(-300)$ & 1 & 4,50 & $\mathrm{C} 2 \mathrm{~d} / \mathrm{P}(120) \mathrm{d} / \psi(-300)$ & 1 & $1740,50 * *$ \\
\hline Resíduo & 33 & 171,50 & Resíduo & 33 & 130,11 \\
\hline C.V. (\%) & & 20,95 & C.V. (\%) & & 31,46 \\
\hline
\end{tabular}

(1) L: Efeito linear. Q: Efeito quadrático. C1 =Ge vs. Ge +Pt; C2: Pt vs. Ge +Pt. *e** significativos a 5 e $1 \%$, respectivamente, pelo testeF. 
capacidade desses fungos em iniciar infeções primárias, comparativamente aos ectomicorrízicos. A redução de $\psi$ acarretou estreitamento da relação MA/ectomi corrizas. Percebeu-semaior queda novalor dessa relação com a redução do $\psi$ de-10 para -40 kPa, em P120 (Quadro 2). Esse estreitamento seria resultante do efeito oposto desempenhado pela redução do $\psi$ sobre col onizações MA eectomicorrízicas. Tal fato evidencia ser a sucessão destes dois tipos de associações influenciada pel as condições ambientais.

\section{Crescimento e absorção de $\mathrm{P}$ pelas plantas}

Plantas de E. grandis crescidas em solo ao qual foram adicionados $120 \mathrm{mg} \mathrm{dm}^{-3}$ de P (P 120) apresentaram maior quantidade de matéria seca (MS), conteúdo de $P$ (CP) e eficiência de utilização do $P$ (EUP) na parte aérea, comparativamente às plantas cultivadas em solo que recebeu a dose de $60 \mathrm{mg} \mathrm{dm}^{-3}$ de P (P60) (Quadros 4 e 5).

A diminuição do $\psi$ reduziu o CP das plantas de E. grandis, da mesma forma que observado por Ruiz

Quadro 4. Matéria seca (MS), conteúdo de P (CP) e eficiência de utilização do $P$ (EUP) na parte aérea de E ucalyptus grandis, considerando as doses de $\mathbf{P}$, os potenciais da água do solo $(\psi)$ e os tratamentos fúngicos

\begin{tabular}{|c|c|c|c|c|c|}
\hline Fósforo & Potencial & $\begin{array}{l}\text { Tratamento } \\
\text { fúngico(1) }\end{array}$ & MS & $\mathbf{C P}$ & EUP \\
\hline $\mathrm{mg} \mathrm{dm} \mathrm{m}^{-3}$ & $\mathrm{kPa}$ & & $\mathrm{g}$ & $\mathrm{mg}$ & $\mathrm{g}^{2} \mathrm{mg}^{-1}$ \\
\hline \multirow[t]{3}{*}{60} & -10 & $\begin{array}{l}\text { Controle } \\
\text { Ge } \\
\mathrm{Pt} \\
\mathrm{Ge}+\mathrm{Pt}\end{array}$ & $\begin{array}{r}9,89 \\
8,62 \\
9,51 \\
10,22\end{array}$ & $\begin{array}{l}7,19 \\
7,14 \\
7,76 \\
8,90\end{array}$ & $\begin{array}{l}15,93 \\
10,55 \\
11,73 \\
11,79\end{array}$ \\
\hline & -40 & $\begin{array}{l}\text { Controle } \\
\text { Ge } \\
\mathrm{Pt} \\
\mathrm{Ge}+\mathrm{Pt}\end{array}$ & $\begin{array}{l}7,78 \\
8,34 \\
7,97 \\
8,29\end{array}$ & $\begin{array}{l}4,92 \\
6,61 \\
7,06 \\
6,58\end{array}$ & $\begin{array}{r}12,36 \\
10,99 \\
9,51 \\
10,46\end{array}$ \\
\hline & -300 & $\begin{array}{l}\text { Controle } \\
\text { Ge } \\
\mathrm{Pt} \\
\mathrm{Ge}+\mathrm{Pt}\end{array}$ & $\begin{array}{l}8,47 \\
9,42 \\
5,99 \\
5,70\end{array}$ & $\begin{array}{l}4,98 \\
5,92 \\
5,48 \\
4,87\end{array}$ & $\begin{array}{c}17,00 \\
16,26 \\
6,68^{(2)} \\
6,81\end{array}$ \\
\hline \multirow[t]{3}{*}{120} & -10 & $\begin{array}{l}\text { Controle } \\
\text { Ge } \\
\mathrm{Pt} \\
\mathrm{Ge}+\mathrm{Pt}\end{array}$ & $\begin{array}{l}15,27 \\
17,15 \\
17,79 \\
19,55\end{array}$ & $\begin{array}{l}13,24 \\
13,54 \\
19,69^{(2)} \\
14,74\end{array}$ & $\begin{array}{l}17,82 \\
22,53 \\
16,85 \\
26,36\end{array}$ \\
\hline & -40 & $\begin{array}{l}\text { Controle } \\
\text { Ge } \\
\mathrm{Pt} \\
\mathrm{Ge}+\mathrm{Pt}\end{array}$ & $\begin{array}{l}12,43 \\
10,30 \\
18,80^{(2)} \\
13,77\end{array}$ & $\begin{array}{c}11,46 \\
9,22 \\
16,95(2) \\
13,84\end{array}$ & $\begin{array}{l}13,78 \\
11,70 \\
21,70 \\
13,95\end{array}$ \\
\hline & -300 & $\begin{array}{l}\text { Controle } \\
\text { Ge } \\
\mathrm{Pt} \\
\mathrm{Ge}+\mathrm{Pt}\end{array}$ & $\begin{array}{r}7,70 \\
9,47 \\
11,05 \\
5,78\end{array}$ & $\begin{array}{l}6,12 \\
6,93 \\
9,50 \\
5,74\end{array}$ & $\begin{array}{r}9,88 \\
13,00 \\
13,47 \\
5,84\end{array}$ \\
\hline
\end{tabular}

${ }^{(1)}$ Ge: Glomus etunicatum. Pt: Pisolithus tinctorius. ${ }^{(2)}$ As médias diferem do controle, não inoculado, pelo teste de Dunnett, a 5\%. et al. (1990) para a soja. Propõe-se que essa redução no CP seja resultante de menor fluxo difusivo de $P$ através do solo, conforme relatado por Bhadoria et al. (1991a,b), e não de um efeito de estresse hídrico sobre a capacidade de absorção de $\mathrm{P}$ pelas raízes; uma vez que as plantas apresentaram metade de suas raízes imersa em um compartimento com solução nutritiva. A redução de CP, em resposta a $\psi$, foi mais acentuada em P120 que em P60 (Quadros 4 e5). Por se tratar de um mesmo solo, a proporção entre o $\mathrm{P}$ adsorvido à fase sólida do solo (fator quantidade) e o P na solução do solo (fator intensidade), ou seja, o fator capacidade é aproximadamente o mesmo nas duas doses de P aval iadas nesteexperimento. Assim, maior quantidade de $\mathrm{P}$ em solução será obtida em P120 e, conseqüentemente, reduções na quantidade deP absorvida pelas raízes, em função da di mi nuição do $\psi$, serão mais drásticas nesta dose do que em P60.

Quadro 5. Análise de variância da matéria seca (MS), conteúdo de $P$ (CP) e eficiência de utilização do $P$ (EUP) na parte aérea de Eucalyptus grandis, considerando as doses de $P$, os potenciais da água do solo $(\psi)$ e os tratamentos fúngicos

\begin{tabular}{|c|c|c|c|c|}
\hline \multirow{2}{*}{$F V^{(1)}$} & \multirow{2}{*}{$\mathbf{G L}$} & \multicolumn{3}{|c|}{ Quadrado médio } \\
\hline & & MS & $\mathbf{C P}$ & EUP \\
\hline $\begin{array}{l}\text { Blocos } \\
\text { P } \\
\psi^{(L)} \text { d/P }(60) \\
\psi^{(Q)} d / P(60) \\
\psi^{(L)} d / P(120) \\
\psi(Q) d / P(120)\end{array}$ & $\begin{array}{l}3 \\
1 \\
1 \\
1 \\
1 \\
1\end{array}$ & $\begin{array}{c}25,46 \\
577,47 * * \\
37,45 \\
1,55 \\
638,87 * * \\
7,83\end{array}$ & $\begin{array}{c}6,25 \\
673,00^{* *} \\
47,40^{*} \\
0,601 \\
542,00^{* *} \\
30,20^{\circ}\end{array}$ & $\begin{array}{c}132,42 \\
365,79^{*} \\
5,27 \\
17,12 \\
855,94^{*} \\
2,06\end{array}$ \\
\hline $\begin{array}{l}C 1 d / P(60) d / \psi^{(-10)} \\
C 2 \quad d / P(60) d / \psi^{(-10)} \\
C 3 d / P(60) d / \psi^{(-10)}\end{array}$ & $\begin{array}{l}1 \\
1 \\
1\end{array}$ & $\begin{array}{l}0,58 \\
1,58 \\
3,55\end{array}$ & $\begin{array}{l}1,66 \\
0,769 \\
5,61\end{array}$ & $\begin{array}{r}62,74 \\
2,78 \\
1,12\end{array}$ \\
\hline $\begin{array}{l}C 1 d / P(60) d / \psi^{(-40)} \\
C 2 d / P(60) d / \psi^{(-40)} \\
C 3 d / P(60) d / \psi^{(-40)}\end{array}$ & $\begin{array}{l}1 \\
1 \\
1\end{array}$ & $\begin{array}{l}0,52 \\
0,27 \\
0,04\end{array}$ & $\begin{array}{r}10,00 \\
0,405 \\
0,173\end{array}$ & $\begin{array}{r}12,48 \\
4,38 \\
0,11\end{array}$ \\
\hline $\begin{array}{l}\text { C1 d/P (60) d/ } / \psi^{(-300)} \\
\text { C2 d/P (60) d/ } \psi^{(-300)} \\
\text { C3 d/P (60) d/ } / \psi^{(-300)}\end{array}$ & $\begin{array}{l}1 \\
1 \\
1\end{array}$ & $\begin{array}{r}6,16 \\
23,53 \\
10,72\end{array}$ & $\begin{array}{l}0,590 \\
0,387 \\
1,84\end{array}$ & $\begin{array}{r}150,52^{\circ} \\
183,55^{\circ} \\
57,90\end{array}$ \\
\hline $\begin{array}{l}C 1 d / P(120) d / \psi^{(-10)} \\
\text { C2 d/P (120) d/ } \psi^{(-10)} \\
\text { C3 d/P (120) } d / \psi^{(-10)}\end{array}$ & $\begin{array}{l}1 \\
1 \\
1\end{array}$ & $\begin{array}{r}25,11 \\
0,81 \\
11,53\end{array}$ & $\begin{array}{l}22,70 \\
75,60^{* *} \\
9,37\end{array}$ & $\begin{array}{r}50,26 \\
64,52 \\
118,63\end{array}$ \\
\hline $\begin{array}{l}\text { C1 d/P(120) d/ } \psi^{(-40)} \\
\text { C2 d/P (120) d/ } \psi^{(-40)} \\
\text { C3 d/P (120) d/ } \psi^{(-40)}\end{array}$ & $\begin{array}{l}1 \\
1 \\
1\end{array}$ & $\begin{array}{c}10,37 \\
144,50 * * \\
1,62\end{array}$ & $\begin{array}{c}10,50 \\
119,00^{* *} \\
1,52\end{array}$ & $\begin{array}{c}12,04 \\
200,00^{\circ} \\
20,16\end{array}$ \\
\hline $\begin{array}{l}C 1 d / P(120) d / / w(-300) \\
C 2 \quad d / P(120) d / w r(-300) \\
C 3 \quad d / P(120) d / w r(-300)\end{array}$ & $\begin{array}{l}1 \\
1 \\
1\end{array}$ & $\begin{array}{c}3,41 \\
4,99 \\
53,52^{\circ}\end{array}$ & $\begin{array}{l}4,84 \\
1,32 \\
1,63\end{array}$ & $\begin{array}{r}2,37 \\
0,44 \\
145,82^{\circ}\end{array}$ \\
\hline Resíduo & 69 & 14,72 & 9,95 & 53,02 \\
\hline C.V. (\%) & & 35,5 & 34,7 & 53,4 \\
\hline
\end{tabular}

(1)L: Efeitolinear. Q: Efeito quadrático. d/: dentro de. C1: Ge, Pt, $\mathrm{Ge}+\mathrm{Pt}$ vs. controle. C2: Ge vs. Pt. C3: Ge, Pt vs. Ge + Pt. $0, * e^{* *}$ significativos a 10,5 e $1 \%$, respectivamente, pelo teste $F$. 
Bhadoria et al. (1991c), avaliando a difusão de P entre porções de solo que receberam $P$ nas doses de 400 e 200 e 200 e $0 \mathrm{mg} \mathrm{kg}^{-1}$, respectivamente, concluíram que, embora o gradiente de $\mathrm{P}$ seja o mesmo nas duas situações, a taxa de difusão foi maior no primeiro caso, em que a concentração do $P$ em solução - ou seja, passível de difusão - foi maior.

A diminuição doCP observada com a redução do $\psi$ resultou em decréscimo significativo, linear, na MS das plantas apenas em P120 (Quadros 4 e 5). Para $\mathrm{P} 60$ o P transportadofoi insuficientepara ocrescimento adequado das plantas, mesmo em - $10 \mathrm{kPa}$. U ma atividade maior de $\mathrm{P}$ na solução do solo em P120 permitiu evidenciar o efeito do potencial hídrico sobre o transporte do nutriente.

De forma semelhante à MS, a EUP diminuiu, linearmente, com a redução do $\psi$ apenas em P120 (Quadros 4 e 5). I sso indicaria que a eficiência de uso do $\mathrm{P}$ seria mais influenciada pelo meio externo quanto maior fosse a disponibilidade do nutriente.

O efeito da colonização micorrízica sobre a produção de biomassa, quando evidenciado, ocorreu apenas em P120 (Quadros 4 e 5). Esses resultados diferem dos apresentados na maior parte dos estudos com micorrizas, que relatam contribuição maior dessas associações em solos com menor disponibilidade de P (Hayman \& Mosse, 1971; Sanders \& Tinker, 1973; Lehto, 1992b). Entretanto, plantas de leucena, com 60 a 90\% das raízes colonizadas por fungos MA, não apresentaram aumento de MS em resposta a essa associação, até que os teores deP na sol ução do sol o fossem el evados a 0,02 mg L-1 (Habte \& F ox, 1993).

Na combinação entre P60 e -300 kPa, na qual a restrição de $P$ foi imposta às plantas nãoapenas pela baixa concentração do nutriente, mas também pela reduzi da difusão em função do menor $\psi$, a avaliação do contraste $\mathrm{Cl}$ ( $\mathrm{Ge}, \mathrm{Pt}, \mathrm{Ge}+\mathrm{Pt}$ versus controle) permitiu concluir que a colonização micorrízica reduziu a EUP, de forma que plantas associadas aos fungos micorrízicos produziram menos MS por $\mathrm{P}$ absorvido. Esta menor EUP pode ser atribuída ao maior custo das associações para as plantas nesta condição de restrição de $P$, na qual, apesar de a taxa de difusão de $\mathrm{P}$ e, conseqüentemente, a absorção destenutrienteterem sido reduzidas pela dimi nuição do $\psi$, observou-se uma colonização fúngica que constituiu um dreno de fotoassimilados para sua manutenção nas raízes. Segundo Aziz \& Habte (1990), a restrição intensa de nutrientes mostrou efeito negativo mais acentuado sobre a efetividade da associação do que sobrea infectividade dos fungos micorrízicos. Essa observação confirma a idéia de comportamento parasítico dos fungos micorrízicos sobre o crescimento das plantas hospedeiras nessas condições (Cooper, 1984).

Pelo contraste C2 (Gevs Pt), em P60 e $\psi-300$ kPa, verificou-se maior efeito depressivo da colonização micorrízica sobrea EUP nas plantas inoculadas com
Pt do que nas com Ge (Quadros 4 e 5). A redução da EUP nesta combinação de menor dose deP e menor $\psi$ ocorreu quando as plantas foram inoculadas com $\mathrm{Pt}$, de forma isol ada ou concomitantemente com Ge. O manto fúngico, presente nas associações ectomicorrízicas e ausente nas MA, constituiu elevado dreno de fotoassimilados (Chilvers \& Harley, 1980), podendo ser a causa da mai or redução da EUP nas plantas colonizadas por Pt. Dessa forma, podese afirmar que, nesta condição de extrema deficiência de $P$, o fungo Pt não foi capaz de incrementar a absorção desse nutriente a ponto de compensar o dreno de carboidratos demandado para seu crescimento e manutenção.

$\mathrm{Na}$ condição de P120 e -10 kPa, a inoculação isolada com Pt incrementou o CP das plantas; contudo, não houve aumento correspondente da MS comparativamente ao controle não inoculado. A combinação entreP 120, -10 kPa e col onização isolada por Pt foi a que proporcionou o maior teor de $\mathrm{P}$ na

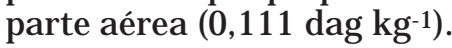

Os demais tratamentos fúngicos não foram capazes de promover alterações significativas no crescimento e absorção de $\mathrm{P}$ pelas plantas, nesta situação. A maior capacidade deabsorção de $P$ pelas raízes associadas a $\mathrm{Pt}$, comparativamente às associadas a Ge, foi atribuída à mai or exploração do sol o pelas hifas do fungo ectomi corrízico em relação às do fungo MA. Em condições de limitação de nutrientes, as hifas de fungos MA podem aumentar a superfície de absor ção das raízes em até 10 vezes, ao passo que as de fungos ectomicorrízicos resultaram em um incremento de até 50 vezes (Siqueira \& Franco, 1988). O aumento da longevidade observado em raízes colonizadas por fungos ectomicorrízicos também contribuiu para a maior absorção de $\mathrm{P}$ em condições de restrição desse nutriente (Bowen, 1973).

Em P120 e $\psi$ de $-40 \mathrm{kPa}$, observou-se aumento do CP acompanhado de incremento na MS das plantas inoculadas com Pt, isol adamente. $\mathrm{O}$ aumento na MS foi resultante não só do incremento do $C P$, mas pode estar relacionadotambém com a tendência observada de elevação da EUP nas plantas colonizadas por $\mathrm{Pt}$, em relação às plantas-controle, já que esta característica apresentou incremento, embora não-significativo estatisticamente, de $57 \%$, em resposta à inoculação com o fungo ectomi corrízico. Comparativamente às plantas inoculadas com Ge, a EUP das plantas associadas a Pt foi significativamente maior, o que significa dizer que a relação custo/ benefício foi menor para plantas col onizadas por Pt, comparativamente às col onizadas por $\mathrm{Ge}$.

Embora, no $\psi$ de $-300 \mathrm{kPa}$, as plantas colonizadas por Pt tenham apresentado valores médios de MS, CP e EUP superiores em 43,5, 55,2 e 36,3\%, respectivamente, aos das plantas-controle, tais diferenças não foram significativas. Não obstante, esses aumentos indicam tendência de a restrição à 
difusão de $\mathrm{P}$, imposta pela redução do $\psi$, ter sido compensada, pelo menos em parte, pela associação com os fungos mi corrízicos. Dessa forma, propõe-se quea redução da umidade, que restringe o transporte deP na solução do sol o, prejudique menos a absorção desse nutriente por raízes colonizadas por fungos micorrízicos. Caberessaltar, além disso, que o efeito da inoculação com Pt na combinação de P120 e $\psi$ de -300 kPa pode ter sido reduzi do, comparativamente à combinação $\mathrm{P} 120$ e-40 kPa, em virtude doaumento da colonização radicular por Pt no menor $\psi$, o que resultaria em maior dreno de carboidratos e, conseqüentemente, em maior custo da associação para a planta. Essa hi pótese pode ser reforçada pela observação de redução da EUP das plantas colonizadas por Pt, isolada ou concomitantemente com Ge, quando o $\psi$ foi reduzi do de-40 para $-300 \mathrm{kPa}$, em P120.

Embora a colonização por fungos micorrízicos tenha apresentado benefícios para as plantas de $\mathrm{E}$. grandis apenas em algumas situações, deve-se atentar para o fato de o método utilizado ter influenciado a resposta das plantas à associação. Segundo diversos autores, a col onização micorrízica pode amenizar os efeitos do déficit hídrico (Nelsen \& Safir, 1982a,b; Lehto, 1992a; Davies et al., 1993) e aumentar a absorção de outros nutrientes de pouca mobilidade no solo, como o zinco (Swaminathan \& Verma, 1979) e o cobre (Gildon \& Tinker, 1983). Desse modo, a eliminação das condições de limitação de água e outros nutrientes, supridos via solução nutritiva, poderia reduzir o efeito da colonização micorrízica sobre o crescimento das plantas. Assim, os benefícios dessas associações, quando evidenciados, devem resultar do aumento na absorção de P.

\section{CONCLUSÕES}

1. Glomus etunicatum colonizou raízes de Eucalyptus grandis em relação direta ao potencial de água dosolo, enquanto Pisol ithus tinctorius respondeu às combinações entre potenciais deágua e doses deP.

2. A inoculação conjunta com $P$. tinctorius e $G$. etunicatum reduziu a ocorrência do primeiro fungo nas raízes de eucalipto e não al terou a do segundo, comparativamente aos tratamentos de inoculação separada de cada fungo.

3. Efeitos positivos deP. tinctorius sobrea absorção de $\mathrm{P}$ e crescimento das plantas foram restritos às combinações de doses de P e potenciais hídricos, nas quais a limitação do fornecimento desse nutriente não foi muito baixa. G. etunicatum não influenciou a absorção de $\mathrm{P}$ e o crescimento das plantas.

4. Os benefícios das associações micorrízicas para as plantas, quando evidenciados, resultaram de aumento na absorção de P.

\section{LITE RATURA CITADA}

AMBLER, J.R. \& YOUNG, J .L. Techniques for determining root length infected by vesicular-arbuscular mycorrhizas. Soil Sci. Soc. Am. J ., 41:551-556, 1977.

AZIZ, T. \& HABTE, M. Stimulation of mycorrhizal activity in Vigna unguiculata through low level fertilization of an oxisol subjected to imposed erosion. Comm. Soil Sci. Plant Anal., 21:493-505, 1990.

BARLEY, K.P. The configuration of the root system in relation to nutrient uptake. Adv. Agron., 22:159-201, 1970.

BHADORIA, P.B.S.; KASELOWSKY, J .; CLAASSEN, N. \& J UNGK, A. Phosphate diffusion coefficient in soil as affected by bulk density and water content. Z. Pflanzenernähr. Bodenk., 154:53-57, 1991a.

BHADORIA, P.B.S.; KASELOWSKY, J .; CLAASSEN, N. \& J UNGK, A. I mpedance factor for chloride diffusion in soil as affected by bulk density and water content. $Z$. Pflanzenernahr. Bodenk., 154:69-72, 1991b.

BHADORIA, P.B.S.; KASELOWSKY, J .; CLAASSEN, N. \& J UNGK, A. Soil phosphate diffusion coefficients: Their dependence on phosphate concentration and buffer power. Soil Sci. Soc. Am. J ., 55:56-60, 1991c.

BOULDIN, D.R. Mathematical description of the diffusion processes in the soil-plant system. Soil Sci. Soc. Am. Proc., 25:476-479, 1961.

BOWEN, G.D. Mineral nutrition of ectomycorrhizae. In: MARKS, G.C. \& KOZLOWSKI, T.T., eds. Ectomycorrhizae: Their ecology and physiology. New York, Academic Press, 1973. p.151-205.

BRAGA, J.M. \& DEFELIPO, B.V. Determinação espectrofotométrica de fósforo em extratos desol o e material vegetal. R. Ceres, 21:73-85, 1974.

BRYLA, D.R. \& DUNIWAY, J .M. Growth, phosphorus uptake, and water relations of sunflower and wheat infected with an arbuscular mycorrhizal fungus. New Phytol., 136:581590, 1997.

CHILVERS, G.A. \& HARLEY, J .L. Visualization of phosphate accumulation in beach mycorrhizas. New Phytol., 84:319326, 1980.

CHILVERS, G.A.; LAPEYRIE, F.F. \& HORAN, D.P. Ectomycorrhizal vs. endomycorrhizal fungi within thesame root system. New Phytol., 107:441-448, 1987.

CLARK, R.B. Characterization of phospatases of intact maize roots. J . Agric. Food Chem., 23:458-460, 1975.

CLARKSON, D.T. Factors affecting mineral nutrient acquisition by plants. Ann. Rev. Plant Physiol., 36:77-115, 1985.

CLARKSON, D.T. \& HANSON, J.B. The mineral nutrition of higher plants. Ann. Rev. Plant Physiol., 31:239-298, 1980.

COOPER, K.M. Physiology of VA mycorrhizal associations. In: POWELL, C.L. \& BAGYARAJ , D. J ., eds. VA Mycorrhiza. Boca Raton, CRC Press, 1984. p.155-186.

CRESS, W.A.; THRONEBERRY, G.D. \& LINDSEY, D.L. Kinetics of phosphorus absorption by mycorrhizal and nonmycorrhizal tomato roots. Plant Physiol., 64:484-487, 1979. 
DANIELS, B.A. \& TRAPPE, J.M. Factors affecting spore germination of the vesicular-arbuscular mycorrhizal fungus, Glomus epigaeus. Mycologia, 72:457-471, 1980.

DAVIES J r., F.T.; POTTER, J .R. \& LINDERMAN, R.G. Drought resistance of mycorrhizal pepper plants independent of leaf $P$ concentration-response in gas exchange and water relations. Physiol. Plant., 87:45-53, 1993.

DIXON, R.K.; GARRET, H.E.; BIXBY, J.A.; COX, G.S. \& THOMPSON, J .G. Growth, ectomycorrhizal development, and root soluble carbohydrates of black oak seedlings fertilizes by two methods. For. Sci., 27:617-624, 1981.

EMPRESA BRASILEIRA DE PESQUISA AGROPECUÁRIA EMBRAPA. Serviço Nacional de Levantamento e Conservação de Solos. Manual de métodos de análise de solo. Rio deJ aneiro, 1997. 212p.

FAQUIN, V.; MALAVOLTA, E. \& MURAOKA, T. Cinética de absorção de fosfato em soja sob influência de micorriza vesículo-arbuscular. R. Bras. Ci. Solo, 14:41-48, 1990.

GILDON, A. \& TINKER, P.B. Interactions of vesicular-arbuscular mycorrhizal infections and heavy metals in plants. II. The effects of infection on uptake of cooper. New Phytol., 95:263$268,1983$.

GIOVANNETTI, M. \& MOSSE, B. An evaluation of techniques for measuring vesicular-arbuscular mycorrhizal infection in roots. New Phytol., 84:489-500. 1980.

HABTE, F. \& FOX, R.L. Effectiveness of VAM fungi in nonsterile soils beforeand after optimization of $\mathrm{P}$ in soil solution. Plant Soil, 151:219-226, 1993.

HAYMAN, D.S. \& MOSSE, B. Plant growth responses to vesiculararbuscular mycorrhiza. I. Growh of Endogoneinoculated plants in phosphate-deficient soils. New Phytol., 71:19-27, 1971.

ITOH, S. \& BARBER, S.A. Phosphorus uptake by six plant species as related to root hairs. Agron. J ., 75:457-461, 1983.

J ACKSON, M.L. Organic matter determination for soil. In: J ACKSON, M.L., ed. Soil chemical analysis. New J ersey, Prentice-Hall, 1958. p.205-226.

KOSKE, R.E. Gigaspora gigantea: observations on spore germination of a VA-mycorrhizal fungus. Mycologia, 73:288300, 1981.

KOSKE, R.E. \& GEMMA, J .N. A modified procedure for staining roots to detect VA mycorrhizas. Mycol. Res., 92:486-505, 1989.

LEHTO, T. Mycorrhizas and drought resistance of Picea sitchensis (Bong.) Carr. I. In conditions of nutrient deficiency. New Phytol., 122:661-668, 1992a.

LEHTO, T. Mycorrhizas and drought resistance of Picea sitchensis (Bong.) Carr. II. In conditions of adequate nutrition. New Phytol., 122:669-669, 1992b.
MARX, D.H.; HATCH, A.B. \& MENDICINO, J .F. High soil fertility decrease sucrose content and susceptibility to loblolly pine roots to ectomycorrhizal infection by Pisolithus tinctorius. Can. J . Bot., 55:1569-1574, 1977.

MEXAL, J . \& REID, C.P.P. The growth of selected mycorrhizal fungi in response to induced water stress. Can. J. Bot., 51:1579-1588, 1973.

MOSSE, B. Plant growth response to vesicular-arbuscular mycorrhiza. IV. In soil given additional phosphate. New Phytol., 72:127-136, 1973.

NELSEN, C.E. \& SAFIR, G.R. Increased drought tolerance of mycorrhizal onion plants caused by improved phosphorus nutrition. Planta, 154:407-413, 1982a.

NELSEN, C.E. \& SAFIR, G.R. The water relations of wellwatered, mycorrhizal and non-mycorrhizal onion plants. J . Am. Soc. Hort. Sci., 107:271-274, 1982b.

OLSEN, S.R.; KEMPER, W.D. \& J ACKSON, R.D. Phosphate diffusion to plant roots. Soil Sci. Soc. Am. Proc., 26:222227,1962

RHODES, L.H. \& GERDEMANN, J.W. Phosphate uptake zones of mycorrhizal and nonmycorrhizal onions. New Phytol., 75:555-561, 1975.

RHEINHEIMER, D.S; ERNANI, P.R.; SANTOS, J.C.P.; KAMINSKI, J . \& KRUNVALD, L. Influência da micorriza no crescimento do Trifol ium riograndensee na predição da absorção de fósforo por um modelo mecanístico. R. Bras. Ci. Solo, 21:191-197, 1997.

RUIZ, H.A.; FERNANDES, B.; NOVAIS, R.F.; ALVAREZV., V.H. \& FERREIRA, P.A. Efeito do conteúdo de água sobre os níveis críticos de fósforo em dois Latossolos. R. Bras. Ci. Solo, 12:43-48, 1988.

RUIZ, H.A.; FERNANDES, B.; NOVAIS, R.F.\& ALVAREZV., V.H. Teor, acúmulo e distribuição de fósforo em plantas de soja em relação ao conteúdo de água do solo. R. Bras. Ci. Solo, 14:181-185, 1990.

SANDERS, F.E. \& TINKER, P.B. Phosphateflow intomycorrhizal roots. Pest. Sci., 4:385-395, 1973.

SANDERS, F.E. The Effect of foliar-applied phosphate on the mycorrhizal infections of onion roots. In: SANDERS, F.E.; MOSSE, B. \& TINKER, P.B., eds. Endomycorrhizas. New York, Academic Press, 1975. p.261-276.

SIDDIQI, M.Y. \& GLASS, D.M. Utilization index: a modified approach to the estimation and comparison of nutrient utilization efficiency in plants. J. Plant Nutr., 4:289-302, 1981.

SIQUEIRA, J.O. \& FRANCO, A.A. Biotecnologia do solo: Fundamentos ePerspectivas. Brasília, MEC-ABEAS-ESALFAEP, 1988. 235p.

SWAMINATHAN, K. \& VERMA, B.C. Responses of three crop species to vesicular-arbuscular mycorrhizal infection on zinc deficient I ndian soils. New Phytol., 82:481-487, 1979. 